\title{
Onfalitis del recién nacido: Infección poco común pero potencialmente letal
}

\section{Omphalitis of the newborn: A rare but life-threatening infection}

\section{Pág. 42,47}

Dra. Constanza Chacón González. Médico general, Costa Rica.

Dra. Silvana Rivera Fumero. Médico general, investigador independiente, Costa Rica.

Dr. Andrey González Chavarría. Médico general, investigador independiente, Costa Rica.

Dr. Esteban Salas Salas. Médico residente de Cirugía General, Hospital Dr. Rafael Ángel Calderón Guardia, San José, Costa Rica.

\section{Resumen}

La onfalitis corresponde a una de las principales causas de mortalidad neonatal en países en vías de desarrollo. Se define como el proceso infeccioso asociado al muñón umbilical, tejido circundante y estructuras adyacentes. Actualmente el manejo adecuado del cordón umbilical permanece controversial, sin embargo, la Organización Mundial de la Salud sugiere el manejo en seco del cordón umbilical en situaciones de bajo riesgo social y el empleo de antisépticos en poblaciones con alta mortalidad neonatal y prevalencia de partos caseros. Tras el diagnóstico, el manejo oportuno consiste en la pronta instauración de antibioticoterapia parenteral con el fin de mitigar posibles eventos adversos.

Palabras clave: Sepsis neonatal; cordón umbilical; infección; recién nacido; clorhexidina

\section{Abstract}

Omphalitis is one of the main causes of neonatal mortality in developing countries. It is defined as the infectious process associated with the umbilical stump, surrounding tissue, and adjacent structures. Currently, adequate management of the umbilical cord remains controversial, however, the World Health Organization suggests dry management of the umbilical cord in situations of low social risk and the use of antiseptics in populations with high neonatal mortality rates and prevalence of home births. After diagnosis, appropriate management consists of the prompt introduction of parenteral antibiotic therapy in order to mitigate possible adverse events.

Key words: Neonatal sepsis; umbilical cord; infection; newborn; chlorhexidine

\section{Introducción}

La onfalitis es un proceso infeccioso del muñón umbilical, tejido circundante y/o estructuras intraabdominales, que ocurre principalmente en el periodo neonatal (1). Es considerado una emergencia pediátrica ya que rápidamente puede progresar a fascitis necrosante, mionecrosis, enfermedad sistémica e inclusive a la muerte (2). La incidencia de onfalitis se encuentra relacionada a la higiene y cuidados del entorno. En países en vías de desarrollo esta entidad puede llegar a afectar hasta el $22 \%$ de los nacimientos domiciliarios y en países desarrollados sólo un $0,7 \%$. La tasa de mortalidad entre todos los lactantes con onfalitis, se estima entre $7 \%$ y $15 \%$, y aumenta hasta cifras de $38 \%$ a $87 \%$ después del desarrollo de fascitis necrosante o mionecrosis (3). El objetivo de esta revisión se centra en exponer los datos disponibles y más recientes sobre la prevención, el diagnóstico y el manejo de esta entidad con el fin de promover su adecuado abordaje.

\section{Métodos}


La búsqueda para la revisión de este tema se hizo en los buscadores como PubMed, UpToDate y GoogleScholar. Se utilizaron lasfrases "omphalitis", "onfalitis neonatal", y acompañada de "etiología", "manejo" y "complicaciones" incluyendo artículos o estudios originales y revisiones de tema. Los criterios de inclusión fueron publicaciones en idiomas inglés, francés y español. Los criterios de exclusión incluyeron artículos publicados en idiomas distintos al español, francés e inglés, con datos no relacionados a onfalitis y el objetivo de esta revisión. Se revisaron 28 artículos que cumplieron con los criterios mencionados. La fecha de la última búsqueda fue el 12 de abril de 2020.

\section{Fisiopatología}

La onfalitis es una infección del cordón umbilical y de los tejidos circundantes, que ocurre principalmente en el período neonatal (4). El cordón umbilical, al ser seccionado, forma un muñón que gradualmente se seca y al caerse cicatriza en cinco a quince días $(4,5)$. En el proceso de cicatrización se forma un espacio entre el cordón umbilical necrosado y la pared abdominal en el cual predomina la presencia de células polimorfonucleares $(4,6)$. Durante la separación normal, se puede acumular tejido que simula material purulento y se diagnostica de manera errónea como infección (6). En estos cinco a quince días el muñón se encuentra en fase de cicatrización por lo que es un sitio de entrada para infección $(6,7)$. Entre mayor la cantidad de días que permanezca el muñón umbilical, mayor la probabilidad de infección ya que los vasos umbilicales se mantienen permeables aproximadamente 20 días $(4,5)$. El tejido desvitalizado del muñón umbilical promueve el crecimiento rápido de estas bacterias, lo que permite la entrada a la circulación sistémica, pudiendo causar infecciones sistémicas severas (6).

\section{Etiología}

En el período neonatal normal, la piel se coloniza de bacterias no patogénicas como Staphylococcus coagulasa negativa y bacilos Diphteroide, pero la piel y bacterias entéricas pueden colonizar el tejido desvitalizado y causar una infección (5, 7). La Organización Mundial de la Salud (OMS), calcula que un cuarto de todas las muertes neonatales son de causa infecciosa, $75 \%$ ocurren en la primera semana de vida, y se considera al cordón umbilical como una vía de entrada (8).

El muñón umbilical justo después del parto se coloniza por múltiples bacterias $(8,9)$. En pocas horas se coloniza por cocos gram positivos y luego se identifica la presencia de múltiples tipos de microorganismos entéricos (7). Se considera que la onfalitis es una infección polimicrobiana, siendo los patógenos más frecuentes Staphylococcus aureus, Streptococcus pyogenes y bacterias gram-positivos como Escherichia coli, Klebsiella pneumoniae y Proteus mirabilis $(4,8,10)$. También se ha descrito la presencia de $S$. aureus meticilino resistente en países con mayor uso de profilaxis antibiótica (11). Si la madre asocia infecciones como corioamnionitis, pueden encontrarse bacterias como Bacteroides fragilis, Clostridium perfringens y Clostridium tetani $(4,6,7)$. Estas mismas bacterias se pueden ver en casos donde se aplique ungüentos herbales, leche humana y cenizas (10).

La onfalitis en ocasiones puede ser una manifestación de una alteración inmunológica, siendo la deficiencia de adhesión leucocitaria (LAD, por sus siglas en inglés) la más frecuente dentro de estas patologías (4). Muchos casos de onfalitis aguda y crónica se han diagnosticado con esta inmunodeficiencia. La LAD presenta una herencia recesiva, donde se asocia leucocitosis, separación tardía del cordón umbilical con o sin onfalitis e infecciones recurrentes (4). La onfalitis también puede ser la manifestación inicial de la 
neutropenia aloinmune neonatal, caracterizada por infecciones cutáneas, neumonía, sepsis y meningitis (4). En raras ocasiones, la causa de la onfalitis puede ser asociada a anormalidades anatómicas como uraco persistente, quistes de uraco o la persistencia del conducto onfalomesentérico (4).

\section{Prevención}

Desde 1998 y hasta la actualidad, la OMS promueve el manejo seco del cordón umbilical, aunado al lavado de manos y correcta higiene, en neonatos nacidos en centros de salud y en comunidades con baja mortalidad neonatal y baja incidencia de onfalitis. Por otro lado, se mantiene que en países en vías de desarrollo, con alta prevalencia de partos caseros y casos frecuentes por esta patología se recomienda el uso tópico de antisépticos con el fin de reducir el riesgo infeccioso $(12,13)$.

El manejo en seco del cordón umbilical consiste en la limpieza inicial de este con jabón neutro y agua tibia, sin la colocación posterior de sustancias adicionales. Se han evaluado distintas medidas de manejo del cordón en el postparto como su cobertura con gaza limpia, exposición al aire evitando que el cordón permanezca en el pañal y evitar la inmersión de este en agua. No obstante, su beneficio no ha sido evaluado de manera objetiva para apoyar su utilidad (14). Además de las medidas de cuidados generales se han utilizado distintos antisépticos con fines preventivos. Entre ellos, sobresale el alcohol de 70 grados y la clorhexidina al $4 \%$ por ser los más utilizados y estudiados; otros corresponden al tinte triple (verde brillante, hemisulfato de proflavina y cristal violeta en una solución acuosa) y antibióticos tópicos como sulfadiazina de plata, tertraciclinas o neomicina $(12,14)$. Sin embargo, se ha demostrado que tanto el tinte triple como los antibióticos tópicos presentan absorción local, especialmente en neonatos prematuros, con posible efecto tóxico sistémico y carcinogénico (5, 12). Asimismo, en países en vías de desarrollo es frecuente el uso de ungüentos y mezclas caseras como aceite de oliva (por su efecto antifúngico), mezcla de hierbas, ceniza, leche materna u otros, no obstante, algunos han demostrado un aumento en el riesgo para infección por Clostridium tetani y se desalienta su uso $(12,14)$.

La clorhexidina, por otro lado, ha demostrado una reducción significativa en la colonización bacteriana del cordón umbilical y según estudios recientes reduce la mortalidad infecciosa en poblaciones de riesgo social $(2,14,15,16)$. Sin embargo, no ha demostrado utilidad en países desarrollados $(13,14,15,16)$, y esto, aunado a que el uso de antisépticos y antibióticos aumenta el tiempo mediano de caída del cordón umbilical (14) e incrementa de manera indirecta el riesgo de desarrollo de onfalitis $(5,12,13)$, se desaconseja la implementación de esta intervención en ausencia de situaciones de alto riesgo infeccioso $(13,14$, 15, 16).

\section{Diagnóstico}

El diagnóstico de la onfalitis es tanto clínico como por datos de laboratorio. Se observa eritema, edema, calor, induración, y en ocasiones secreciones purulentas en la zona del muñón umbilical $(7,9,11,12,17,18,19)$. También puede haber olor fétido de la secreción y hemorragia umbilical por retraso en la trombosis de los vasos umbilicales $(6,20)$.

Otras veces pueden no existir síntomas o signos localizados y presentarse más bien con datos de sepsis, como es la fiebre, letargia y cambios en la alimentación $(4,5,6,9,12,19,21,22)$ Es por esto que se deben hacer los laboratorios pertinentes al neonato para descartar una infección sistémica o sepsis. Estos incluyen: hemoleucograma con énfasis en conteo neutrofílico y plaquetario,

\section{(9) $\mathcal{( 0 ) \Theta}$}


hemocultivos, cultivos de secreciones, punción lumbar, electrolitos, pruebas de función renal, pruebas de función hepática, gases arteriales, urianálisis, urocultivo, y reactantes de fase aguda (proteína $\mathrm{C}$ reactiva y procalcitonina) $(4,21)$.

Según la severidad de afectación de la infección, se puede estadiar en cuadro grados. El grado 1 incluye funisitis (o infección del tejido conectivo umbilical) con secreción purulenta que puede o no ser fétida. El grado 2 incluye las características del grado 1 más celulitis de la pared abdominal. El grado 3 abarca tanto la afectación local como sistémica, con datos de sepsis, coagulación intravascular diseminada, shock, e incluso falla multiorgánica. $\mathrm{Y}$ por último, el grado 4 adiciona complicaciones como equimosis, bulas y crépitos en la pared abdominal, con extensión a las facias superficiales y profundas y el músculo $(4,23)$.

Estudios complementarios como el ultrasonido, la tomografía computarizada y la resonancia magnética pueden usarse como apoyo para descartar complicaciones o diagnósticos diferenciales, discutidos enseguida $(4,20)$.

\section{Diagnóstico Diferencial}

El diagnóstico de onfalitis puede confundirse con otras patologías del cordón umbilical, sus orígenes embriológicos, y anomalías anatómicas o inmunológicas. A nivel local se debe considerar la funisitis, que es una infección localizada al muñón umbilical sin afectación de vasos umbilicales o estructuras adyacentes. La hernia umbilical es una estructura que protruye a través del ombligo, es reducible, y suele resolver con el tiempo; es infrecuente que se presente con infección. Los pólipos umbilicales son masas que pueden estar presentes debido a remanentes onfalomesentéricos. Secreciones intestinales (biliares o fecales) deben hacer sospechar una fístula enteroumbilical. Otros remanentes onfalomesentéricos incluyen quistes, senos, o el divertículo de Meckel. Una secreción clara, amarillenta, sin datos purulentos, puede ser sugestivo de un uraco persistente. Tejido rojorosado que permanezca en el ombligo posterior a la caída del cordón, con secreción serosa o serosanguinolenta, es altamente sugestivo de un granuloma umbilical. El sangrado recurrente a través del muñón umbilical debe hacer sospechar trastornos de la coagulación. En casos de onfalitis recurrentes, que asocien prolongación en la caída del cordón u otras infecciones recurrentes, y/o cultiven agentes infrecuentes (como Citrobacter sp.), se deben sospechar inmunodeficiencias primarias linfocitarias, asociadas a neutrófilos, o por deficiencia de adhesión leucocitaria, que se confirmarán con sus estudios específicos $(4,6$, $11,19,20,21,24,25)$.

\section{Tratamiento}

Se recomienda terapia antimicrobiana de amplio espectro de manera parenteral por un mínimo de 10 días, dependiente al estado del paciente y su respuesta inicial al tratamiento $(6,20)$. Dado que los microorganismos más frecuentes corresponden a patógenos Gram-positivo y Gram-negativo, la elección del agente debe ser efectivo contra estos (20). El tratamiento empírico inicial, previo a resultado del cultivo, consiste en el uso de penicilina antiestafilocócica y aminoglucósidos con el fin de reducir el riesgo de complicaciones como sepsis y fascitis necrotizante. En zonas de alta prevalencia de MRSA (Staphylococcus aureus meticilino resistente) se debe considerar sustitución de penicilina por vancomicina $(6,20)$. Además, ante la sospecha de microorganismos anaerobios, en especial en presencia de descarga purulenta fétida, o en neonatos nacidos de madres con corioamnionitis se sugiere la implementación de clindamicina o metronidazole $(6,20)$. Actualmente se desaconseja el uso de antibióticos orales o tópicos como bacitracina o mupiracina, así como el uso de alcohol en pacientes con síntomas leves para el manejo de onfalitis, debido a la información insuficiente para sustentar su uso $(20,26)$. No obstante, en países en vías de desarrollo con un sistema de salud deficitario y con escasez de 
recursos para el ingreso del infante se permite el uso de cotrimoxazole, penicilina o cefalexina oral y aminoglucósidos intramusculares, durante al menos 7 a 10 días (26).

\section{Complicaciones}

Se debe de reconocer de manera temprana la onfalitis para prevenir complicaciones sistémicas y severas de esta enfermedad (8). La sepsis es la complicación más frecuente y puede progresar a shock séptico y muerte $(8,27)$. Otras complicaciones raras, peroconaltamorbimortalidad son: peritonitis, gangrena intestinal, evisceración del intestino delgado, absceso hepático, arteritis umbilical séptica y trombosis de la vena portal (27, 28). A continuación, se describen dos de estas complicaciones que usualmente requieren de resolución quirúrgica.

\section{Peritonitis}

Es secundario a la extensión directa de la infección a la cavidad abdominal. Puede presentarse como íleo adinámico que falla al tratamiento usual $(7,27,28)$. Usualmente se debe realizar un ultrasonido de abdomen para identificar abscesos intraabdominales y se debe abordar con laparotomía y drenaje. Las arterias umbilicales pueden contener material purulento por lo que se deben resecar para controlar el proceso infeccioso $(27,28)$.

\section{Fascitis necrotizante}

Se ha reportado en $13.5 \%$ de los neonatos con onfalitis $(6,27)$. Usualmente inicia como eritema y celulitis, el cual progresa a la formación de microabscesos y sin tratamiento evoluciona a la fascitis necrotizante $(27,28)$. El sitio más frecuente es la pared abdominal anterior, pero puede extenderse a los genitales externos y el tórax $(9,28)$. Usualmente es polimicrobiana de rápida progresión, con toxicidad sistémica y mala respuesta a antibioticoterapia inicial $(8,9)$. En estos casos se debe de agregar cobertura antibiótica de amplio espectro, profilaxis antitetánica si la madre no recibió la vacuna durante el embarazo y consultar al servicio de cirugía para valorar la necesidad de debridación quirúrgica $(9,28)$. La tasa de mortalidad de la fascitis necrotizante puede alcanzar hasta $60-85 \%$, siendo mayor en los casos que no se realizó debridación quirúrgica $(9,27)$.

\section{Conclusión}

La onfalitis neonatal es una patología infecciosa, que usualmente se presenta con eritema y/o edema del muñón umbilical, afectando desde la piel circundante hasta, en casos de mayor severidad, el tejido subcutáneo, fascia, músculo e inclusive potencial invasión sistémica. El diagnóstico temprano en el periodo neonatal, ante sospecha clínica y por confirmación con estudios complementarios, permite mitigar complicaciones asociadas a esta patología.

\section{Referencias}

1.World Health Organization. WHO Recommendations on Postnatal Care of the Mother and Newborn [Internet]. 2014. WHO Press; Geneva. [Consultado 11 Abril 2020]; Disponible en: https://www.who.int/maternal_child_adolescent/ documents/postnatal-care-recommendations/en/

2.Hodgins S. Chlorhexidine and Newborn Omphalitis and Mortality [Internet]. Lancet Glob Health. 2017; 5(3): e270-e271.[Consultado 11 Abril 2020]; Disponible en: https:// www.pubmed.ncbi.nlm.nih.gov/28193393

3.Stewart D, Benitz W. Umbilical Cord Care in the Newborn Infant [Internet]. Pediatrics. 2016; 130(3): e20162149. [Consultado 12 Abril 2020]; Disponible en: https://pubmed. ncbi.nlm.nih.gov/27573092

4.Gallagher PG, Shah SS. Omphalitis: Background, Pathophysiology, Etiology [Internet]. Emedicine.medscape. com. 2020 [cited 10 April 2020]. Available from: https:// emedicine.medscape.com/article/975422-overview

5.Zupan J, Garner P, Omari AAA. Topical umbilical cord care at birth. Cochrane Database of Systematic Reviews. 2004; 3 : CD001057. 
6.Painter K, Feldman J. Omphalitis. [Actualizado 2019 junio 4]. In: StatPearls [Internet]. Treasure Island (FL): StatPearls Publishing; 2020 enero. Disponible en: https://www.ncbi.nlm. nih.gov/books/NBK513338/

7.Manikoth P, George M, Vaishnav A, Sajwani MJ. Omphalitis. Lancet. 2004; 364: 1522.

8.World Health Organization. Newborns: reducing mortality [Internet]. Who.int. 2020 [citado 10 Abril 2020]. Disponible en: https://www.who.int/news-room/fact-sheets/detail/newbornsreducing-mortality

9.Brook, I. Cutaneous and subcutaneous infections in newborns due to anaerobic infections. J Perinat Med. 2002; 30: 197-208.

10.Sinha A, Sazawal S, Pradhan A, Ramji S, Opiyo N. Chlorhexidine skin or cord care for prevention of mortality and infections in neonates. Cochrane Database of Syst. Rev. 2015.

11.Sengupta M, Banerjee S, Banerjee P, Guchhai P. Outstanding Prevalence of Methicillin Resistant Staphylococcus aureus in Neonatal Omphalitis. JCDR. 2016; 10(9): DM01-DM03.

12.Senturk Erenel A, Gulsen V, Yaman Efe S, Semiha O, Ozgen S, Erenoglu R. Comparison of Olive Oil and Dry-Clean Keeping Methods in Umbilical Cord Care as Microbiological. Matern Child Health J. 2010; 14: 999-1004.

13.Imdad A, Mullany L, Baqui A, Arifeen S, Tielsch J, Khatry S, et al. The effect of umbilical cord cleansing with chlorhexidine on omphalitis and neonatal mortality in community settings in developing countries: a meta-analysis. BMC Public Health 2013; 13(Suppl 3): S15. Disponible en: http://www. biomedcentral.com/1471-2458/13/S3/S15

14.Leante-Castellanos J, Pérez-Muñuzuri A, Ruiz-Campillo C, Sanz-López E, Benavente-Fernández I, Sánchez-Redondo $\mathrm{M}$, et al. Recommendations for the care of the umbilical cord in the newborn. An Pediatr (Barc). 2019; 90(6): 401.e1-401. e5. Disponible en: https://www.sciencedirect.com/science/ article/pii/S234128791930078X

15.Mullany L, Arifeen S, Khatry S, Katz J, Shah R, Baqui A, et al. Impact of Chlorhexidine Cord Cleansing on Mortality, Omphalitis, and Cord Separation Time Among Facility-Born Babies in Nepal and Bangladesh. Pediatr Infect Dis J. 2017; 36(10): 1011-1013. doi:10.1097/INF.0000000000001617.

16.Sankar M, Chandrasekaran A, Ravindranath A, Agarwal R, Paul VK. Umbilical cord cleansing with chlorhexidine in neonates: a systematic review. J Perinatol. 2016; 36 (Suppl 1): S12-20. doi: 10.1038/jp.2016.28.
17.Mir F, Sundar Tikmani S, Shakoor S, Javed Warraich $\mathrm{H}$ et al. Incidence and etiology of omphalitis in Pakisan: a community-based cohort study. J Infec Dev Ctries. 2011; 5(12): 828-833.

18.Beasely SW. Umbilicus and its extensive clinical repertoire. J Paediatr Child Health. 2017; 53: 1123-1126.

19.Muniraman, H., Sardesai, T., \& Sardesai, S. Disorders of the Umbilical Cord. Pediatrics in Review. 2018; 39(7): 332341.

20.Palazzi DL, Brandt ML. Care of the umbilicus and management of umbilical disorders. UpToDate [Internet]. Actualizado el 2 de abril 2020, consultado el 11 de abril 2020.

21.Kliegman RM, St Geme JW, Blum NJ, Shah SS, Tasker RC, Wilson KM. Nelson Textbook of Pediatrics. 21 edición. Pensilvania: Elsevier Inc.; 2020.

22.Cushing AH. Omphalitis: a review. Pediatr Infect Dis. 1985; 4(3): 282-285

23.Sawardekar KP. Changing spectrum of neonatal omphalitis. Pediatr Infect Dis J. 2004; 23:22-26.

24.Cuvelier P, Bouteiller A, Fortpied P, Lonneux M, Blumental S. Omphalite a Streptococcus pyogenes et cellulite du pied chez un nourrisson de 11 jours. Rev Med Brux. 2018; 169171.

25.Sivathanu S, Sampath S, Sridhar I. Recurrent Omphalitis and Nonhealing Ulcers in a 7-month-old Girl. Pediatrics in Review. 2016; 37(11): 491-493

26.Qamar, F., Tikmani, S., Mir, F., Zaidi, A. Community-based management and outcome of omphalitis in newborns in Karachi, Pakistan. Journal of Pakistan Medical Association. 2013; 63(11): 1364-1369. Disponible en: https://ecommons. aku.edu/pakistan_fhs_mc_women_childhealth_paediatr/442

27.López-Medina M, López-Araque A, Linares-Abad M, López-Medina I. Umbilical cord separation time, predictors and healing complications in newborns with dry care. PLoS ONE. 2020;15(1):e0227209.

28.Ameh E, Nmadu P. Major complications of omphalitis in neonates and infants. Pediatr Surg Int. 2002;18(5-6):413416. 